\title{
Lower Bispectral index values in psychiatric patients: A prospective, observational study
}

\author{
Venkatapura J. Ramesh, Muthu C. Radhakrishnan, Rohini Thimmaiah', Kesavan Muralidharan', \\ Jagadisha Thirthalli', Ganne S. Umamaheshwara Rao, Bangalore N. Gangadhar ${ }^{1}$
}

\begin{abstract}
Background: Bispectral index score (BIS) is a processed electroencephalographic parameter used to measure level of sedation in anaesthetised patients. In few studies of psychiatric patients undergoing electroconvulsive therapy (ECT), it was observed that the BIS values were lower at baseline. It is not clear from those studies whether the BIS values are really low. Also, it is not clear whether the lower values are related to the primary psychiatric illness or the due to the effect of ECT. Therefore, we studied the BIS values in psychiatric illnesses and compared them with the normal controls. Materials and Methods: BIS index was recorded in 237 patients with various psychiatric illness (Group P) and 40 control patients without any psychiatric illness undergoing spinal surgery (Group C). BIS values were recorded in supine position before breakfast and before the morning doses of antipsychotic/benzodiazepine medications. It was recorded during resting state in all the subjects. Results: BIS values were lower in group P compared to control group (a mean of $89.8 \pm 7.8$ vs $95.7 \pm 2.4, P<0.000 \mathrm{I}$ ). In the group $P$, the patients with psychosis and bipolar disorder had significantly lower BIS values than the patients with depression $(P=0.04)$. Conclusions: BIS values in psychiatric patients are lower than those in the control group. Psychotic and bipolar disorders are associated with significantly lower BIS values than the depression.
\end{abstract}

Key words: Bipolar disorder, bispectral index, depression, diazepam, monitoring, psychiatric illness

\section{INTRODUCTION}

The Bispectral index (BIS) monitoring was initially developed to assess the depth of hypnosis during general anaesthesia and to prevent awareness under anaesthesia ${ }^{[1]}$ Later, it was used in various other conditions like assessment of adequacy of sedation in various clinical situations, monitoring the depth of coma, titration of barbiturate coma and detection of cerebral ischaemia. ${ }^{[2-6]}$ Several other potential applications are under exploration.

\begin{tabular}{|l|l|}
\hline \multicolumn{2}{|c|}{ Access this article online } \\
\hline Quick Response Code: & Website: \\
\hline & www.jnaccjournal.org \\
\cline { 2 - 2 } & \\
\hline & \\
\hline
\end{tabular}

Electroencephalographic changes have been documented in patients with psychiatric illness. Baseline EEG in patients with dementia has been reported to show an increase in slow-wave activity and this has been demonstrated as low awake BIS values. ${ }^{[7]}$ Most studies of BIS in psychiatric illness have been in the context of electroconvulsive therapy (ECT).$^{[8,9]}$ These studies used BIS to titrate the depth of anaesthesia with the intention of achieving adequate hypnosis as well as an adequate duration of seizure. In these studies, low BIS values have been noted in psychiatric patients in the awake state (baseline). We also observed in few patients that the baseline BIS values were lower in patients scheduled for ECT. It is not clear if the disease per se causes a decrease in BIS or it is due to the effect of ECT. Also, it would be of interest to see if BIS values are different across various psychiatric illnesses. We designed the current investigation to assess the baseline BIS values in varied psychiatric patients in a

Departments of Neuroanesthesia, and ${ }^{1}$ Psychiatry, National Institute of Mental Health and Neuro Sciences, Bangalore, Karnataka, India

Address for correspondence:

Dr. Venkatapura J Ramesh, Department of Neuroanesthesia, National Institute of Mental Health and Neurosciences, Hosur Road,

Bangalore - 560 029, Karnataka, India. E-mail: drvjramesh@gmail.com 
prospective, observational study. The aim of the study was to record the BIS values in patients diagnosed with psychosis (comprising schizophrenia, acute psychosis, unspecified psychoses, schizo affective disorders), bipolar disorder (manic, depressive or mixed states) and major depression (including recurrent depressive disorder) and compare with the normal controls. Our secondary aim was to compare the BIS values in different psychiatric illness groups.

\section{MATERIALS AND METHODS}

The study was undertaken in a tertiary care university hospital for psychiatric and neurological illness. Institutional ethics committee approval was obtained and after a written informed consent, subjects were enrolled into the study over a period of one year. In such cases where the subject was not in a position to give informed consent because of the severity of illness (such as catatonia), consent was obtained from the caretaker.

\section{Subjects}

The study included two groups - patients with psychiatric illnesses admitted to the hospital (group P) and a control group (group C). Group P included consecutive patients admitted to hospital with ICD-10 diagnoses ${ }^{[10]}$ of schizophrenia (F20.0-F20.9), acute psychosis (F23.0-F23.9), unspecified psychosis (F29), schizoaffective disorder (F25), bipolar disorder (F30.0-F31.9) and major depression (F32.0-F33.9). Patients with clinically identifiable central nervous system (CNS) illness and mental retardation were excluded from the study. Patients who have received ECT in the past 2 months were also excluded. The control group comprised preoperative neurosurgical patients scheduled for spinal surgeries. These patients did not have any intracranial pathology and did not receive any form of sedation/anaesthesia in the previous 4 days.

\section{Assessments}

The demographic and clinical details of the patients were recorded. Medication history was noted in all patients. The BIS values were recorded by using a BIS monitor (A-2000, Aspect Medical Systems, Newton, USA) before breakfast and before the morning dose of antipsychotic/benzodiazepine medications with the subjects lying in supine position. The BIS sensor strip was applied on to the right side of the forehead after cleaning the skin with alcohol. The patients were instructed to relax with the eyes closed lightly. This was done to decrease the artifacts due to blinking of eyelids. A signal quality index of 50 was considered as a minimum requirement to record the BIS values. Two minutes after application of the electrodes, two consecutive BIS values were recorded at an interval of 1 minute. The two BIS values were averaged for analysis.

\section{Statistical analysis}

The averaged BIS values were compared between the groups. Student's $t$-test and analysis of variance (ANOVA) tests were used for analyzing the parametric data. Least significant difference (LSD) was used for post hoc multiple comparisons. Chi-square test/Fisher's exact test were used for analyzing the non-parametric data. The significance for $P$-value was fixed at $<0.05$. The data was analysed using Statistical Package for the Social Sciences (SPSS) version 16.0.

\section{RESULTS}

The study comprised 237 psychiatric patients in group $\mathrm{P}$ and 40 patients in the control group. The demographic characteristics of the patients were comparable [Table 1]. The SQI was $>60$ in $85 \%$ of the patients (Interquartile $25-75: 67-90$ ). The BIS values were significantly lower in group $\mathrm{P}(89.8 \pm 7.8)$ compared to group C $(95.7 \pm 2.4)$.

An attempt was made to compare the BIS values in three major diagnostic categories namely psychosis, bipolar disorders and depression. The other diagnoses were not included in the analysis as there were very few patients under each diagnostic category. When BIS values were compared across the three major diagnostic categories, the values were significantly lower in psychotic and bipolar disorders in comparison with patients with depression [Table 2]. This difference was significant even after controlling for the sedative effects of the medications (both sedation equivalents of antipsychotic medications and diazepam equivalents of benzodiazepine medications, $P<0.002) .{ }^{[11,12]}$ The benzodiazepine group of drugs were used more in depressive disorders and antipsychotic medications were used more in psychiatric and bipolar disorder patients [Table 3].

Table 1: Demographic data

\begin{tabular}{llcc}
\hline & Group P $(\boldsymbol{n}=\mathbf{2 3 7})$ & $\begin{array}{c}\text { Group C } \\
(\boldsymbol{n}=\mathbf{4 0})\end{array}$ & P value \\
\hline Age (Y)* & $31 \pm 9.7$ & $36 \pm 11.5$ & 0.004 \\
Sex (M:F) & $109: 128$ & $22: 18$ & 0.3 \\
Diagnosis & Organic disorders-16 & & \\
& Psychotic disorders-110 & & \\
& Bipolar disorders-72 & & \\
& Depressive disorders-29 & & \\
& Others-10 & & \\
& $89.8 \pm 7.8$ & $95.7 \pm 2.4$ & 0.001 \\
BIS values* & $(88.8-90.8)$ & $(94.9-96.3)$ & \\
&
\end{tabular}

*Values are mean \pm standard deviation. Values in parentheses are 95\% confidence intervals. BIS = Bispectral index score 


\section{DISCUSSION}

The main findings of our study are: 1) BIS values in psychiatric patients are significantly lower in comparison with those in the control patients; 2) Patients with psychosis and bipolar disorder have lower BIS values than patients with depression.

Till date, no study has assessed BIS as the primary outcome measure in patients with psychiatric illnesses. There are a few studies which have documented low BIS values in patients undergoing ECT. Ochai $\mathrm{R}$ et al., ${ }^{[8]}$ studied patients with severe depressive illness coming for ECT. The recorded baseline BIS was $89 \pm 6$. All the patients were on medications. But this study concerned seizure inducibility during ECT and included only 16 patients, with evaluation of 100 ECT sessions. It is not clear from the methodology, whether the baseline was calculated only from the first 16 sessions or from the baselines of all the sessions. An earlier publication has reported that interictal BIS values are decreased by ECT. ${ }^{[13]}$ Inclusion of baseline values of all sessions to calculate the study baseline will decreases the baseline BIS values artificially. Hanss et al., ${ }^{[14]}$ studied 14 medically resistant major depressive patients who underwent 109 ECT sessions. The baseline BIS was 93 (range 76-99). Here again, all the sessions were included in calculating the baseline. To our knowledge, ours is the first major study to examine the BIS values in a large population of psychiatric patients with all major diagnostic categories, independent of the ECT treatment.

The patients with psychosis and bipolar disorder had significantly lower BIS values than patients with depression. This difference may be due to the disease itself or it may be due to the difference in the type of medications that they were receiving. There are no

\section{Table 2: BIS values in various diagnostic categories}

\begin{tabular}{lcccc}
\hline & $\begin{array}{c}\text { Psychotic } \\
\text { disorders } \\
(\boldsymbol{n}=\mathbf{1 1 0})\end{array}$ & $\begin{array}{c}\text { Bipolar } \\
\text { disorders } \\
(\boldsymbol{n}=\mathbf{7 2})\end{array}$ & $\begin{array}{c}\text { Depressive } \\
\text { disorders } \\
(\boldsymbol{n}=\mathbf{2 9})\end{array}$ & $\boldsymbol{P}$ value \\
\hline BIS & $89.4 \pm 8.7$ & $90.0 \pm 7.1$ & $93.2 \pm 5.8$ & 0.04 \\
values & $(87.7-91.0)$ & $(87.3-90.7)$ & $(91.0-95.4)$ & \\
\hline
\end{tabular}

All values are mean \pm standard deviation. Values in parenthesesare $95 \%$ confidence intervals. Post hoc multiple comparison (LSD): Depresion Vs psychotic $P=0.02$, depresion Vs bipolar $P=0.016$. BIS = Bispectral index score characteristic EEG patterns described for psychiatric illness. But slowing of EEG with delta waves has been described in the psychiatric patients. ${ }^{[15,16]}$ It is known that benzodiazepines cause marked increase in beta-activity on the electroencephalogram. ${ }^{[17]}$ This increased beta-activity in the frontal region could have been reflected as the lower BIS values (light sedation) ${ }^{[18]}$ Antipsychotic medications can also cause diffuse slowing of the EEG ${ }^{[19]}$ This might have also resulted in lower BIS values. The current study is not designed to assess the relative contribution of the two classes of drugs to the changes in the EEG and its consequent impact on the BIS values. Future studies which are specifically aimed at addressing this question will be able to clarify this.

The BIS values in normal individuals are around 95-100. It will be in the range of $60-90$ in patients who are sedated; the BIS being inversely related to the degree of sedation. In clinically anaesthetised patients it will be in the range of 40-60. However, it has been noted that in psychiatric patients it can be as low as 60-70 and the patients can be awake without any clinical manifestation of sedation. ${ }^{[8,9,14]}$ Anaesthesiologists have to take this into consideration while assessing the depth of anaesthesia in such patients. This raises an important question as to at what level of BIS score, the psychiatric patients are adequately anaesthetised. Probably we have to use the clinical methods for assessing the depth of anaesthesia in these patients instead of the EEG-based monitors. There are a few studies which advocate a BIS of 50-55 in patients undergoing ECT to have adequate depth of anaesthesia without a significant effect on the seizure duration. ${ }^{[8]}$ But with interictal BIS values being affected by ECT itself, BIS values considered adequate at one point may not be appropriate for subsequent ECT sessions during the later part of the treatment. ${ }^{[13]}$

The merit of this study is that the age group of the patients is relatively low. Also, there were no patients with dementia. Therefore, the effects of aging and any dementia (subclinical) are automatically eliminated. The BIS values are known to be low in the elderly and in dementic patients. ${ }^{[9]}$ One limitation of the study is that there was a statistically significant difference in the age between the two groups. The control group patients are older by about a mean of 5 years. This difference of 5 years between the groups in adult patients is unlikely to cause any significant difference in the BIS values,

Table 3: The pattern of medication usage in various psychiatric illnesses

\begin{tabular}{lcccc}
\hline & $\begin{array}{c}\text { Psychotic disorders } \\
(\boldsymbol{n}=\mathbf{1 1 0})(\mathbf{0})\end{array}$ & $\begin{array}{c}\text { Bipolar disorders } \\
(\boldsymbol{n}=\mathbf{7 2})(\mathbf{\%})\end{array}$ & $\begin{array}{c}\text { Depressive } \\
\text { disorders }(\boldsymbol{n}=\mathbf{2 9})(\mathbf{\%})\end{array}$ & $\boldsymbol{P}$ value \\
\hline Benzodiazepines & $31(28.2)$ & $36(50)$ & $19(65.5)$ & 0.001 \\
Antipsychotics & $101(93.5)$ & $62(86.1)$ & $15(51.7)$ & 0.001 \\
\hline
\end{tabular}

Many patients have received combination of both the groups of medications 
though it cannot be ruled out. Another limitation is that the number in the control group was not equal to study group. However, our aim was also to compare different groups of psychiatric patients and we presumed some groups may be lower and some groups will be higher than 40 patients. Also, the awake BIS values in the normal adult population are a well-known phenomenon. Thus, we kept the control group at a reasonable number of 40 patients.

\section{CONCLUSIONS}

BIS values in psychiatric patients are lower than in non-psychiatric control patients. The BIS values in psychosis and bipolar disorder were significantly lower than in depression. Future studies should address the issue of the causes for these differences. Anaesthesiologists have to take the above facts into consideration while assessing the depth of anaesthesia using the BIS monitor in patients with psychiatric illness.

\section{REFERENCES}

1. Johansen JW, Sebel PS. Development and clinical application of electroencephalographic bispectrum monitoring. Anesthesiology 2000;93:1336-44.

2. Liu J, Singh H, White PF. Electroencephalographic bispectral index correlates with intraoperative recall and depth of propofol-induced sedation. Anesth Analg 1997;84:185-9.

3. Gajraj RJ, Doi M, Mantzaridis H, Kenny GN. Analysis of the EEG bispectrum, auditory evoked potentials and the EEG power spectrum during repeated transitions from consciousness to unconsciousness. Br J Anaesth 1998;80:46-52.

4. Schnakers C, Ledoux D, Majerus S, Damas P, Damas F, Lambermont B, et al. Diagnostic and prognostic use of bispectral index in coma, vegetative state and related disorders. Brain Inj 2008;22:926-31.

5. Cottenceau V, Petit L, Masson F, Guehl D, Asselineau J, Cochard JF, et al. The use of bispectral index to monitor barbiturate coma in severely brain-injured patients with refractory intracranial hypertension. Anesth Analg 2008;107:1676-82.

6. Estruch-Pérez MJ, Barberá-Alacreu M, Ausina-Aguilar A, Soliveres-Ripoll J, Solaz-Roldán C, Morales-Suárez-Varela MM. Bispectral index variations in patients with neurological deficits during awake carotid endarterectomy. Eur J Anesthesiol 2010;27:359-63.

7. Renna M, Handy J, Shah A. Low baseline Bispectral Index of the electroencephalogram in patients with dementia. Anesth Analg 2003;96:1380-5.

8. Ochiai R, Yamada T, Kiyama S, Nakaoji T, Takeda J. Bispectral index as an indicator of seizure inducibility in electroconvulsive therapy under thiopental anesthesia. Anesth Analg 2004;98:1030-5.

9. Gunawardane PO, Murphy PA, sleigh JW. Bispectral index monitoring during electroconvulsive therapy under propofol anesthesia. Br J Anaesth 2002;88:184-7.

10. The ICD-10 Classification of mental and behavioural disorders. Clinical descriptions and diagnostic guidelines. Geneva: World Health Organization; 1992.

11. Miller DD. Atypical antipsychotics: Sleep, sedation, and efficacy. Prim Care Companion J Clin Psychiatry 2004;6:3-7.

12. Ashton $\mathrm{H}$. The treatment of benzodiazepine dependence. Addiction 1994;89:1535-41.

13. Thimmaiah R, Thirthalli J, Ramesh VJ, Radhakrishnan MC, Muralidharan $\mathrm{K}$, Mahadevaiah $\mathrm{M}$, et al. Effect of a course of electroconvulsive therapy on interictal bispectral index values: A prospective study. J ECT 2012;28:20-3.

14. Hanss R, Bauer M, Bein B, Goeder R, Buttgereit B, Schulz-Du Bois AC, et al. Bispectral index-controlled anesthesia for electroconvulsive therapy. Eur J Anesthesiol 2006;23:202-7.

15. Sengoku A, Takagi S. Electroencephalographic findings in functional psychoses: State or trait indicators? Psychiatry Clin Neurosci 1998;52:375-81.

16. Binnie CD, Prior PF. Electroencephalography. J Neurol Neurosurg Psychiatry 1994;57:1308-19.

17. Gotman J, Gloor P, Quesney LF, Olivier A. Correlations between EEG changes induced by diazepam and the localization of epileptic spikes and seizures. Electroencephalogr Clin Neurophysiol 1982;54:614-21.

18. Bennett C, Voss LJ, Barnard JP, Sleigh JW. Practical use of the raw electroencephalogram waveform during general anesthesia: The art and science. Anesth Analg 2009;109:539-50.

19. Boutros NN. Diffuse electroencephalogram slowing in psychiatric patients: A preliminary report. J Psychiatry Neurosci 1996;21:259-63.

How to cite this article: Ramesh VJ, Radhakrishnan MC, Thimmaiah R, Muralidharan K, Thirthalli J, Umamaheshwara Rao GS, et al. Lower Bispectral index values in psychiatric patients: A prospective, observational study. J Neuroanaesthesiol Crit Care 2014;1:121-4.

Source of Support: Nil, Conflict of Interest: None declared.

\section{Staying in touch with the journal}

1) Table of Contents (TOC) email alert

Receive an email alert containing the TOC when a new complete issue of the journal is made available online. To register for TOC alerts go to www.ijoy.org.in/signup.asp.

\section{2) RSS feeds}

Really Simple Syndication (RSS) helps you to get alerts on new publication right on your desktop without going to the journal's website. You need a software (e.g. RSSReader, Feed Demon, FeedReader, My Yahoo!, NewsGator and NewzCrawler) to get advantage of this tool. RSS feeds can also be read through FireFox or Microsoft Outlook 2007. Once any of these small (and mostly free) software is installed, add www.ijoy.org.in/rssfeed.asp as one of the feeds. 